\title{
Nanopartículas e aplicações endodônticas: uma revisão da literatura
}

\author{
Nanoparticles and endodontics applications: a literature review
}

\begin{abstract}
Iris Sol Figueiredo Telles, ${ }^{1}$ Maíra do Prado, ${ }^{1}$ Renata Antoun Simão ${ }^{1}$
'Programa de Engenharia Metalúrgica e de Materiais, Universidade Federal do Rio de Janeiro, RJ, Brasil

- Os autores declaram que não há conflito de interesse.
\end{abstract}

\section{Resumo}

Objetivo: este artigo pretende apresentar uma revisão da literatura quanto ao conhecimento científico disponível sobre a aplicação de nanopartículas na Endodontia. Material e Métodos: foram avaliadas as seguintes propriedades: atividade antimicrobiana, prevenção contra formação de biofilmes bem como sua eliminação, compatibilidade, resistência ao envelhecimento, resistência mecânica, dispersão para as regiões de anatomia complexa e regeneração dos tecidos. Foi realizada uma pesquisa bibliográfica de estudos publicados entre 2004 e junho de 2017, coletados através das bases de dados SciELO e Medline. Resultados: um total de 50 artigos foi selecionado. A maior parte dos estudos que avalia a atividade antimicrobiana mostra resultados positivos em relação a incorporação das nanopartículas. A maior parte dos estudos que avalia a atividade antimicrobiana mostra resultados positivos em relação a incorporação das nanopartículas nos materiais. Os que avaliaram as propriedades mecânicas e estruturais dos materiais encontraram aumento na rigidez, microdureza e uma menor porosidade, que esta relacionada com aumento da resistência destes materiais. Quando adicionadas aos materiais, a maioria dos estudos conclui que não foram modificadas as propriedades e quando houveram modificações, causaram um efeito positivo. Quanto à biocompatibilidade, pode haver um aumento da toxicidade em altas concentrações de nanopartículas, no entanto, outros estudos afirmam biocompatibilidade e propriedades osteogênicas, sendo esta característica associada ao tipo de nanopartícula. Conclusão: concluiu-se que as nanopartículas estão até o presente momento sendo avaliadas em nível laboratorial, com resultados promissores para práticas clínicas futuras. Palavras-chave: Endodontia; Nanoestruturas; Nanopartículas.

\section{AbstRact}

Objective: the purpose of this article was to present a review of the literature on available scientific knowledge about the application of nanoparticles in endodontics. Material and Methods: the following properties were evaluated: antimicrobial activity, prevention against biofilms formation as well as their elimination, compatibility, resistance to aging, mechanical resistance, dispersion to complex anatomy regions and tissue regeneration. A bibliographic research of studies was performed from 2004 to june 2017, collected through the SciELO and Medline databases. Results: a total of 50 articles were selected. Most of the studies that evaluated the antimicrobial activity showed positive results regarding the incorporation of nanoparticles. Most of the studies that evaluated the antimicrobial activity showed positive results regarding the incorporation of nanoparticles in the materials. Those that evaluated the mechanical and structural properties of the materials, found an increase in stiffness, microhardness, and a lower porosity, which is related to increased resistance of these materials. When added to materials, most studies conclude that the properties were not modified and when there were adjustments, they caused a positive effect. Regarding biocompatibility, there are an increase in toxicity in high concentrations of nanoparticles, however, other studies affirm biocompatibility and osteogenic properties, being this characteristic associated with the type of nanoparticle. Conclusion: it was concluded that nanoparticles are currently being evaluated at the laboratory level, with promising results for future clinical practices. Keywords: Endodontic; Nanostructures; Nanoparticles.

\section{Introdução}

A nanotecnologia é um novo campo na ciência que vem crescendo nas últimas décadas. Trata-se de uma tecnologia promissora e inovadora, por projetar novos materiais com novas funções e também conceder àqueles existentes melhorias nas suas propriedades. ${ }^{1,2} \mathrm{Na}$ engenharia de materiais, na química e na indústria farmacêutica, esta ciência já tem seu campo consolidado e continua em grande expansão. ${ }^{1,-5}$ É considerado um nanomaterial aquele que, após sua síntese, apresenta partículas que atinjam dimensões entre 1 e 100 nanômetros (nm). ${ }^{1,3,6,7}$

O conjunto de aplicações dos nanomateriais na Endodontia abrangem transporte de fármacos, ${ }^{8-10}$ estratégias antimicrobianas, ${ }^{2,5,8-38}$ propriedades mecânicas, ${ }^{2,38,39-45}$ e propriedades osteogênicas. ${ }^{10,46,47}$ Porém, há o desafio científico de formular nanopartículas (NPs) que ao serem adicionadas aos produtos comerciais existentes não alterem substancialmente suas propriedades biológicas, físico-químicas e mecânicas. ${ }^{2}$ Outra condição importante é a segurança biológica, para que sejam aplicadas sem aumentarem os efeitos citotóxicos, e de preferência com efeitos colaterais mínimos. $^{4,38,39,48-50}$

Este artigo tem como objetivo fazer uma revisão da literatura científica publicada sobre o uso das nanopartículas na área da Endodontia, bem como descrever as diferentes novas estratégias terapêuticas testadas nos estudos.

\section{Material e Métodos}

A revisão da literatura foi realizada por meio de consulta às bases de dados bibliográfica SciELO via Bireme e Medline via PubMed. A estratégia de busca foi através da inserção de DeCS (Descritores e Ciências da Saúde) e termos livres, como "Endodontia", "Nanomateriais", "Nanopartículas" e "Nanotecnologia". Foram selecionados 50 artigos entre 2004 e junho de 2017, dentre revisões de literatura e artigos científicos, que abordaram os tópicos de interesse para esta revisão.

Rev. Bras. Odontol., Rio de Janeiro, v. 74, n. 2, p. 167-75, abr./jun. 2017 
Foram selecionados artigos que avaliaram as nanopartículas de prata, quitosana, polietilenimina de amônio quaternário, agregado trióxido mineral branco, vidro bioativo, óxido de zinco, poli (ácido láctico-co-ácido glicólico) e clorexidina.

\section{Revisão de Literatura}

Após a seleção dos artigos, as informações relevantes foram extraídas de cada estudo e abordadas nessa revisão. Para fins didáticos esta revisão foi subdividida em relação ao tipo de nanopartícula avaliada.

\section{- Nanopartículas de Prata}

As nanopartículas de prata (NPsAg) consistem em nano estruturas formadas a partir de íons de prata. A prata tem sido utilizada como medicamento por anos devido suas propriedades antimicrobianas, porém seu emprego foi diminuído com a descoberta dos antibióticos. ${ }^{7}$ Quando as NPsAg surgiram, elas demonstraram potente propriedade anti-inflamatória e antimicrobiana, tendo interações especiais com bactérias e fungos. Com isso tornaram-se muito populares sendo utilizadas em diversas aplicações médicas. ${ }^{5}$

$\mathrm{Na}$ Odontologia, as NPsAg são encontradas na literatura incorporadas aos materiais dentários, como resina composta, resina acrílica, implantes e materiais endodônticos. ${ }^{7}$ Para o uso endodôntico são propostas como: medicações, associadas aos cimentos endodônticos e soluções irrigadoras, como recobrimento de materiais obturadores e como conservantes. Estas aplicações devem-se principalmente às propriedades antimicrobianas. ${ }^{5,11-20}$

Neste contexto, soluções de NPsAg são consideradas irrigantes endodônticos em potencial, uma vez que, devido à grande relação área-volume, o efeito antimicrobiano das NPsAg é muito maior que a sua forma sólida. ${ }^{5}$ Lotfi et al., ${ }^{11}$ utilizando concentração de $0,005 \%$ de NPsAg, verificaram resultados antimicrobianos semelhantes ao $\mathrm{NaOCl} 5,25 \%$. Os mesmos resultados foram encontrados por Moghadas et al. ${ }^{12}$ e por González-Luna et al. ${ }^{13}$ No entanto, Wu et al..$^{14}$ concluíram, através da análise de micrografias de biofilmes na dentina radicular, que a irrigação com as NPsAg não modificou a estrutura do biofilme, tendo efeito semelhante ao soro e inferior ao $\mathrm{NaOCl} 2 \%$.

A atividade antimicrobiana contra E. faecalis do gel de NPsAg nas concentrações $0,05 \%, 0,1 \%$ e $0,2 \%$ foi testada em comparação a clorexidina (CHX) $2 \%$ e ao fenol canforado. Não foram encontradas diferenças significativas entre NPsAg $0,05 \%$ e as outras substâncias, porém o gel de NPsAg $0,1 \%$ e $0,2 \%$ foi mais eficaz que a CHX e fenol canforado. ${ }^{15}$ Ainda, Bruniera et al. ${ }^{16}$ adicionaram NPsAg a 3 veículos, hidroxietilcelulose, carbomer e polietilenoglicol e verificaram que os veículos experimentais incorporados com NPsAg apresentaram atividade antimicrobiana e produziram formulações estáveis, sendo o hidroxietilcelulose àquele que apresentou melhores propriedades físicas.

Estudos apontaram que NPsAg podem ser utilizadas como medicação intracanal em associação ao hidróxido de cálcio $\left[\mathrm{Ca}(\mathrm{OH})_{2}\right]$, potencializando o seu efeito antibacteriano. ${ }^{17,18}$ Os resultados encontrados por Javidi et al. ${ }^{17} \mathrm{e}$ Afkhamia et al.${ }^{18}$ mostraram que o número de colônias observadas após o tratamento com $\mathrm{Ca}(\mathrm{OH})_{2}$ com NPsAg foi significativamente menor que o observado com $\mathrm{Ca}(\mathrm{OH})_{2}$ puro. No entanto, divergem de Alabdulmohsen \& $\mathrm{Saad}^{5}$ que mostraram que o $\mathrm{Ca}(\mathrm{OH})_{2}$ resultou em maior porcentagem de redução bacteriana do que as NPsAg. O efeito antibacteriano das NPsAg foi menor que o $\mathrm{Ca}(\mathrm{OH})_{2}$ sozinho ou quando combinados.

A ação antimicrobiana também foi avaliada em relação à incorporação de NPs nos materiais obturadores. Cimento de Agregado Trióxido Mineral (MTA) com NPsAg (1\% MTA/ NP) possui maior ação antimicrobiana quando comparado ao cimento obturador de MTA não modificado. ${ }^{19} \mathrm{~A}$ infiltração de corante e microinfiltração bacteriana foi avaliada em cones de guta-percha convencional e cones revestidos com NPsAg. Embora os resultados encontrados tenham sido estatisticamente semelhantes, os autores concluem que usar a guta-percha revestida pode ser mais eficaz para tratamentos endodônticos. ${ }^{20}$

Nevarez-Rasconb et al. ${ }^{39}$ propuseram o uso de pontas de fibras de vidro revestidas com NPsAg como alternativa aos cones de guta-percha. Os resultados indicaram que as pontas alcançaram rigidez e resistência, sendo este material promissor para uso clínico uma vez que possui propriedades antibacterianas através da prata, transmissão de luz pela fibra óptica, o que possibilita fotopolimerizar resinas fluidas no canal, além de garantir a compatibilidade com pinos de fibra de vidro e resinas.

A aglomeração e o tamanho das NPsAg são responsáveis pela citotoxicidade, que foi estuda em associação com três cimentos endodônticos, AH Plus, Epiphany e GuttaFlow, sendo este último composto por nanopartículas de prata que funcionam como conservantes. Os resultados mostraram citotoxicidade aumentada com o tempo independente do material. A toxicidade do GuttaFlow foi atribuída à liberação de NPsAg. ${ }^{48}$

Em resposta tecidual de ratos, a biocompatibilidade foi avaliada com soluções de $1 \mathrm{~mL}$ de NPsAg-47ppm, NPsAg $-23 \mathrm{ppm}$, e $\mathrm{NaOCl} 2,5 \%$. Os resultados indicaram que as soluções de NPsAg foram biocompatíveis, principalmente em 
concentrações mais baixas. ${ }^{49} \mathrm{O}$ efeito de um irrigante composto por NPsAg analisado sobre células como fibroblastos de ratos e células-tronco primárias do ligamento periodontal humano, não foi citotóxico para as células testadas tanto em ensaio de contato direto como em contato indireto. ${ }^{50}$ Agentes iônicos imidazólios com NPsAg positivamente carregadas exibiram menor toxicidade quando comparados ao $\mathrm{NaOCl}$ e CHX. Além disso, os autores afirmam que a carga superficial das NPsAg é importante na eficácia bactericida. ${ }^{4}$

\section{- Nanopartículas de Quitosana}

As nanopartículas de quitosana (NPsCS) são formadas por partículas de quitosana (poly[1,4],-b-D-glucopyranosamine), que é um biopolímero catiônico não tóxico, que possui propriedades antibacterianas. ${ }^{6,21}$ São utilizadas na medicina para tratamento de diversas doenças sistêmicas e na Odontologia são aplicadas sobre biofilmes bacterianos e na cicatrização de lesões, devido às suas propriedades antimicrobianas e biocompatibilidade. ${ }^{6}$ As NPsCS possuem características interessantes para aplicação clínica futura, tais como: cor (branca), custo, disponibilidade e facilidade de modificação. ${ }^{21}$

$\mathrm{Na}$ Endodontia, as NPsCS são relatadas como tendo baixos níveis de citotoxicidade, com eficaz eliminação bacteriana, quando associadas aos cimentos endodônticos e soluções irrigantes. ${ }^{21-28}$ Outra característica relevante é a presença de efeito antimicrobiano mesmo após um longo tempo de exposição, ${ }^{21,22}$ como nos estudos de Shrestha et al. ${ }^{21}$ que com concentrações de 2, 5 e $10 \mathrm{mg} / \mathrm{mL}$ de NPsCS eliminaram totalmente o biofilme de E. faecalis em 8-12 horas de interação. Após 90 dias, as NPsCS ainda mostravam inibição bacteriana.

Quando incorporadas nos cimentos ThermaSeal Plus e MTA Fillapex, aumentaram a ação antibacteriana destes que permaneceram mesmo após 4 semanas. ${ }^{22} J a ́$ para o cimento Grossman tipo 801 com NPsCS, os resultados foram diferentes, pois as NPsCS apresentaram maior atividade antimicrobiana quando isoladas, porém essa atividade era diminuída quando adicionadas ao cimento. ${ }^{23}$

Além de cimentos, a incorporação de NPsCS em pastas de $\mathrm{Ca}(\mathrm{OH})_{2}$ foi estuda por Del Carpio-Perochena et al., ${ }^{24}$ onde houve uma redução significativa nas colônias de E. faecalis com exposição a curto e longo prazo.

Novas estratégias antibacterianas, tais como a terapia fotodinâmica (PDT) tem sido comparada e/ou somada às NPsCS. Ao avaliar a influência de inibidores teciduais comuns sobre o efeito antibacteriano de NPsCS e PDT, os resultados indicaram que ambas as técnicas tiveram sua ação antimicrobiana inibida. Os autores concluíram que os componentes da polpa e albumina de soro bovino podem interagir com as NPs e a presença destes inibidores pode ter dificultado a absorção de fotoiniciadores na célula bacteriana. Modificações e melhorias destas técnicas devem ser estudadas antes de considerá-las para aplicação clínica. ${ }^{25}$ Em uma pesquisa que avaliou a formação de biofilmes na interface cimento-dentina, os canais radiculares receberam tratamentos superficiais como: CS fosforilado, conjugado com CS Rosa bengala e irradiação fotodinâmica ou uma combinação de ambos. Os canais foram obturados com cimentos incorporados de NPsCS e os resultados indicaram que o cimento com NPs inibiu a formação de biofilmes na interface, efeito encontrado em todos os tratamentos. ${ }^{26}$ Estudos mostram que o uso de fotoiniciadores funcionalizados com NPsCS alcançou eficácia antibacteriana e exibiu uma penetração mais profunda na estrutura do biofilme o que resultou em danos significativamente aumentados na membrana bacteriana. ${ }^{27,28}$

$\mathrm{O}$ uso de um ultrassom focalizado de alta intensidade (high-intensity focused ultrasound - HIFU) foi proposto em associação com as NPsCS com o objetivo de melhorar a desinfecção dos canais radiculares, nos túbulos dentinários, através da ação antimicrobiana das NPsCS. Foi possível obter penetração de até $1.000 \mu \mathrm{m}$ das NPsCS, sendo este um método potencial para o fornecimento de NPs nos túbulos dentinários. ${ }^{6}$

\section{- Nanopartículas de Polietilenimina de Amônio Quaternário}

Nanopartículas de polietilenimina de amônio quaternário (NPsQPEI) são partículas poliméricas insolúveis, carregadas positivamente, ${ }^{47}$ que têm gerado interesse na medicina moderna por proporcionarem uma superfície bactericida de largo espectro e um efeito superior ao encontrado em antibióticos convencionais. ${ }^{33}$ NPsQPEI vem sendo incorporadas em cimentos provisórios, resinas compostas e cimentos endodônticos, devido ao seu efeito antimicrobiano contra bactérias orais sem comprometer as propriedades físicas dos materiais. $^{2,29-33}$

Quando incorporadas aos cimentos AH Plus e GuttaFlow houve uma inibição total do crescimento bacteriano, além disso, a análise de microscopia eletrônica de varredura indicou dano e lise da parede celular bacteriana. ${ }^{29}$ Resultados semelhantes foram encontros por Barros et al. ${ }^{30}$ que testaram o AH Plus e Pulp Canal Sealer EWT na forma comercial, não modificada, ou contendo as NPs. Os autores observaram uma melhora na capacidade antimicrobiana com a adição de NPs. Em outro estudo com os mesmos cimentos endodônticos os resultados evidenciaram que os cimentos 
não modificados perderam grande parte dos efeitos antibacterianos após 7 dias. As propriedades físico-químicas e mecânicas dos cimentos afetadas pela incorporação de NPsQPEI, como tempo de fixação, molhabilidade e potencial zeta, podem aumentar a atividade antibacteriana e aumentar a capacidade do cimento em penetrar no sistema de canais radiculares. ${ }^{2}$

As NPsQPEI, quando incorporadas em uma pequena porcentagem em cimentos à base de resina epóxi, produzem um potente efeito antibacteriano. ${ }^{31} \mathrm{Em}$ análises de superfície, incrustações de NPsQPEI sobre o cimento à base de resina epóxi, atraiu ativamente as bactérias, causando desestabilização e morte bacteriana. Em conclusão as NPsQPEI mostraram atividade antimicrobiana contra E. faecalis. ${ }^{32}$ Beyth et al. ${ }^{33}$ obtiveram resultados que indicaram que as NPsQPEI interagem com a membrana celular das bactérias em poucos minutos, desencadeando a morte celular por meio de variações de $\mathrm{pH}$, mas ao mesmo tempo impedindo a penetração de NPs em células eucarióticas, deste modo, impedindo um possível efeito citotóxico. Além disso, foram mantidas as propriedades físicas dos cimentos à base de resina epóxi testados.

NPsQPEI 2\% isoladas não afetaram o comportamento celular, porém incorporadas aos cimentos AH Plus e Pulp Canal Sealer modulam a proliferação e diferenciação de células ósseas, sem aumentar a citotoxicidade destes. ${ }^{46} \mathrm{Em}$ outro estudo que também avaliou NPsQPEI 1\% adicionadas aos cimentos AH Plus, Epiphany e GuttaFlow, a citotoxicidade não foi aumentada e as propriedades básicas dos cimentos não foram alteradas. ${ }^{47}$

\section{- Nanopartículas de Agregado Trióxido Mineral Branco}

As nanopartículas de agregado trióxido mineral branco ou white mineral trioxide aggregate (NWMTA) são partículas de agregado trióxido mineral (MTA), um cimento que vem sendo utilizado principalmente pela capacidade de manter o pH elevado e pela liberação de íons cálcio, proporcionando atividade antibacteriana e regeneração dos tecidos. $^{40}$

$\mathrm{Na}$ Endodontia, a versão nano modificada é usada com a promessa de tornar a presa do cimento MTA mais rápida e com resistência a ambientes ácidos. Um aumento da área superficial do pó pode reduzir o tempo de presa e aumentar a microdureza mesmo em ambientes de $\mathrm{pH}$ ácido após a hidratação. ${ }^{40,42}$ Analisando o efeito do $\mathrm{pH}$ sobre a solubilidade do cimento MTA branco e cimento de NWMTA, devido às suas pequenas partículas e seus diferentes aditivos, o cimento de NWMTA foi capaz de produzir uma menor porosida- de no material, o que resultou em uma maior resistência a ambientes ácidos. ${ }^{43}$ WMTA e NWMTA mostraram maior resistência após exposição a forças nano-compressivas em comparação ao Bioaggregate. ${ }^{44}$

Um estudo que avaliou a influência de Metronidazol, Minociclina e Ciprofloxacina (misturados ou isolados) e de CHX, sobre a força de adesão e microdureza superficial de cimentos de MTA com diferentes tamanhos de partícula, obteve superior resistência de união e microdureza com o cimento de NPs. Este também resistiu mais do que o MTA convencional ao efeito das soluções de antibióticos. ${ }^{41}$

\section{- Nanopartículas de Vidro Bioativo}

$O$ vidro bioativo ou bioactive glass (BAG) é um material nanoparticulado osteoindutor, com propriedades antibacterianas. Consiste em $\mathrm{SiO}_{2}, \mathrm{Na}_{2} \mathrm{O}, \mathrm{CaO}_{2}$ e $\mathrm{P}_{2} \mathrm{O}_{5}$ em diferentes concentrações em soluções aquosas e libera seus compostos iônicos ao longo do tempo. ${ }^{3}$ A liberação de íons $\mathrm{Na}^{+}$e $\mathrm{Ca}^{2+}$ do vidro corroído e a incorporação de prótons $\mathrm{H}_{3} \mathrm{O}^{+}$no vidro elevam o $\mathrm{pH}$ do ambiente tornando-o hostil para micro -organismos. ${ }^{34}$ Além disso, a sílica liberada tem sido relacionada ao efeito antibacteriano e a indução da mineralização da dentina. Para aumentar a propriedade antibacteriana, algumas pesquisas estão se concentrando em diminuir o tamanho de partícula de vidro e assim aumentar a troca ativa da superfície do vidro e do líquido circundante. ${ }^{1}$

Devido à sua capacidade de criar um ambiente alcalino, ensaios com o pó de BAG mostraram forte efeito antimicrobiano, ${ }^{34}$ eliminando a infecção após 5 dias com resultados superiores ao $\mathrm{Ca}(\mathrm{OH})_{2} \cdot{ }^{35}$ No entanto, outros estudos observaram um efeito antibacteriano superior do $\mathrm{Ca}(\mathrm{OH})_{2}$ após 10 dias e após 50 dias de inspeção. ${ }^{36,37}$

Mohn et al. ${ }^{45}$ desenvolveram materiais compósitos utilizando poliisopreno (PI) ou policaprolactona (PCL) incorporados de BAG 45S5, na tentativa de criar uma interface de vedação de hidroxiapatita e, assim, tornar desnecessário o uso de um cimento endodôntico. Os autores verificaram que a incorporação de BAG melhorou a capacidade de vedação imediata dos compósitos desenvolvidos.

\section{- Nanopartículas de Óxido de Zinco}

Nanopartículas de óxido de zinco (NPsZnO) são formadas por nanoparticulados metálicos, conhecidos por causarem peroxidação lipídica pelas espécies reativas de oxigênio (reactive oxygen species - ROS), como superóxido $\left(\mathrm{O}_{2}-\right)$ e radicais hidroxila $(\mathrm{OH})$. O contato direto ou indireto destas com a bactéria podem causar danos à membrana, devido a toxicidade das ROS. ${ }^{25} \mathrm{O} \mathrm{ZnO}$ é um material seguro que tem sido amplamente utilizado em aplicações biomédicas como no tratamento do câncer. ${ }^{38}$ 
As aplicações de NPsZnO na Endodontia está associada às propriedades antibacterianas. ${ }^{21,23,38}$ Além disso, possuem vantagens como biocompatibilidade, custo-benefício e disponibilidade. ${ }^{21} \mathrm{~A}$ adesão de E. faecalis na dentina tratada com estas NPs tem sido reduzida. As NPsZnO isoladas possuem menor potencial zeta e menor atividade antimicrobiana contra $E$. faecalis, quando comparadas as outras NPs testadas como as NPsCS. Porém essa atividade é aumentada quando são misturadas ao cimento de óxido de zinco, provavelmente devido à sua maior compatibilidade com o pó do cimento. ${ }^{21,23}$ Rad et al. ${ }^{38}$ produziram cimentos de NPsZ$\mathrm{nO}$ e NPsZnO:Ag, que foram comparados com cimento de óxido de zinco e eugenol e cimento AH26. O pó sintetizado com as NPs apresentou melhores propriedades estruturais, impediram a microinfiltração e aumentaram a atividade antibacteriana.

\section{- Nanopartículas de Poli (ácido láctico-co-ácido glicólico)}

As nanopartículas de poli (ácido láctico-co-ácido glicólico) ou NPs de PLGA são partículas poliméricas usadas como sistemas de liberação controlada. Na Endodontia apenas um estudo testou o encapsulamento de drogas fotoativas para aplicação da terapia fotodinâmica, que tem sido uma promissora técnica antimicrobiana adjuvante no tratamento endodôntico. ${ }^{8}$ Foi sugerido o encapsulamento do azul de metileno em NPs de PLGA como um novo sistema de liberação de fármacos com efeito foto destrutivos promissores na eliminação de biofilmes dos canais radiculares. Canais infectados com $E$. faecalis foram tratados com estas NPs que se encontravam concentradas nas paredes celulares dos micro-organismos e com o sinergismo da luz foram capazes de reduzir as colônias bacterianas. ${ }^{8}$

\section{- Nanopartículas de Clorexidina}

As nanopartículas de clorexidina (CHX) são sistemas de nanoencapsulados que geralmente envolvem um revestimento polimérico que carregam em seu interior o fármaco CHX. Desta maneira a CHX tem sua eficácia terapêutica maximizada devido ao maior controle da biodisponibilidade e liberação do fármaco no local desejado. Estudos buscaram liberação da CHX por meio de NPs na tentativa de manter a inibição bacteriana no interior dos canais radiculares. ${ }^{9,10}$ Quando dispersas em matriz de hidrogel composto por 1\% de hidroxietilcelulose há maior inibição bacteriana durante períodos mais longos de tempo. Essas características classificaram as NPs de CHX como um excelente material para a manutenção da descontaminação intracanal. ${ }^{9}$

Apesar da CHX ser um potente antibacteriano contra $E$. faecalis, é ineficaz na indução da mineralização dos tecidos do periápice radicular. NPs de silicato de cálcio mesoporoso são biomateriais recentemente sintetizados com excelente capacidade para promover a mineralização e liberar moléculas bioativas de forma controlada. As NPs de silicato de cálcio mesoporoso apresentaram a capacidade de liberar CHX e também $\mathrm{Ca}^{2+} \mathrm{e} \mathrm{SiO}_{3}{ }^{2-}$, com baixa citotoxicidade, produzindo excelente atividade antimicrobiana e de mineralização in vitro. Este material foi proposto como uma nova medicação intracanal ou como um novo material de preenchimento de defeito ósseo para defeitos infectados. ${ }^{10}$

\section{Discussão}

Em virtude do pequeno tamanho alcançado, as NPs podem ser levadas em regiões de anatomias complexas. Características físico-químicas aprimoradas, tais como tamanhos ultrapequenos, grande proporção superfície/massa e maior reatividade química podem produzir efeitos antimicrobianos superiores como resultado de uma interação aumentada com as células microbianas. ${ }^{3,11}$ Outra característica importante é a capacidade das nanopartículas permanecerem com atividade antimicrobiana mesmo após um longo tempo de ação. ${ }^{2,9,18,21,22}$

A aplicação bem-sucedida das NPs, quando utilizadas como irrigantes ou medicações, dependerá da eficácia e do método de administração utilizado para dispersar essas partículas nas complexidades anatômicas do sistema de canais radiculares, que em geral são regiões não instrumentadas. ${ }^{6,22,27}$ É importante que as NPs tenham tamanho e morfologia adequados para poderem adentrar nos túbulos dentinários, os quais apresentam diâmetros médios de 0,5 - $4 \mu \mathrm{m}$, uma vez que a permanência bacteriana nos túbulos dentinários tem sido associada a infecção persistente do canal radicular. ${ }^{3,9,31}$

$\mathrm{Na}$ presente revisão, ao todo foram selecionados 50 artigos que descreveram a relevância do uso das NPs na Endodontia (Tabela 1). Não foi encontrado qualquer teste clínico em humanos (in vivo) ou ainda estudos clínicos randomizados. Quando as nanopartículas foram adicionadas aos materiais endodônticos, as propriedades destes não foram alteradas, e quando houve modificações, causaram um efeito sinérgico positivo.

Rev. Bras. Odontol., Rio de Janeiro, v. 74, n. 2, p. 167-75, abr./jun. 2017 
Tabela 1. Resumo dos achados obtidos nos trabalhos que avaliaram o uso de nanopartículas e sua incorporação aos materiais endodônticos

\begin{tabular}{|c|c|c|c|}
\hline Autores & Nanopartícula & Propriedades Avaliadas & Resultados \\
\hline Lotfi et al. ${ }^{11}$ & NPsAg & Ação antimicrobiana & $\mathrm{NPs}=\mathrm{NaOCl} 5,25 \%$ \\
\hline Moghadas et al. ${ }^{12}$ & NPsAg & Ação antimicrobiana & $\mathrm{NPs}=\mathrm{NaOCl} 5,25 \%$ \\
\hline González-Luna et al. ${ }^{13}$ & NPsAg & Ação antimicrobiana & $\mathrm{NPs}=\mathrm{NaOCl} 2,25 \%$ \\
\hline Wu et al. ${ }^{14}$ & NPsAg & Ação antimicrobiana & $\mathrm{NaOCl} 2 \%>\mathrm{NPs}$ \\
\hline Bo e Kayombo ${ }^{15}$ & NPsAg & Ação antimicrobiana & NPs $0,1 \%$ e de $0,2 \%>C H X 2 \%$ e fenol canforado \\
\hline Bruniera et al. ${ }^{16}$ & NPsAg & Ação antimicrobiana & $\begin{array}{l}\text { NPs + hidroxietilcelulose ou carbomer ou polietileno- } \\
\text { glicol, apresentaram atividade antimicrobiana }\end{array}$ \\
\hline Javidi et al.17 & NPsAg & Ação antimicrobiana & $\mathrm{Ca}(\mathrm{OH}) 2+\mathrm{NPs}$, reduziu os micro-organismos \\
\hline Afkhamia et al. ${ }^{18}$ & NPsAg & Ação antimicrobiana & $\mathrm{Ca}(\mathrm{OH}) 2+\mathrm{NPs}$ eficaz mesmo após 1 semana \\
\hline Alabdulmohsen e Saad ${ }^{5}$ & NPsAg & Ação antimicrobiana & NPs $<\mathrm{Ca}(\mathrm{OH}) 2$ sozinho ou quando combinados \\
\hline Samiei et al. ${ }^{19}$ & NPsAgw & Ação antimicrobiana & Cimento de MTA + NPs > MTA \\
\hline Shantiaee et al. ${ }^{20}$ & NPsAg & Ação antimicrobiana & Guta-percha + NPs = guta-percha convencional \\
\hline Nevarez-Rasconb et al. ${ }^{39}$ & NPsAg & Mecânicas & $\begin{array}{l}\text { Fibras de vidro ópticas + NPs: rigidez, resistência, } \\
\text { melhor fotopolimerização e compatibilidade }\end{array}$ \\
\hline Bouillaguet et al. ${ }^{48}$ & NPsAg & Citotoxicidade & Maior citotoxicidade com o tempo \\
\hline Gomes-Filho et al. $4^{9}$ & NPsAg & Citotoxicidade & NPs biocompatíveis \\
\hline Chan et al..$^{50}$ & NPsAg & Citotoxicidade & NPs não foi citotóxico para as células \\
\hline Abbaszadegan et al ${ }^{4}$ & NPsAg & Citotoxicidade & $\begin{array}{l}\text { Agentes iônicos imidazólios + NPs positivamente } \\
\text { carregadas < } \mathrm{NaOCl} \text { e } \mathrm{CHX}\end{array}$ \\
\hline Shrestha et al. ${ }^{21}$ & NPsCS & Ação antimicrobiana & NPs com inibição bacteriana mesmo após 90 dias \\
\hline Del Carpio-Perochena et al.22 & NPsCS & Ação antimicrobiana & $\begin{array}{l}\text { NPs + ThermaSeal Plus e NPs + MTA Fillapex, maior } \\
\text { ação antimicrobiana mesmo após } 4 \text { semanas }\end{array}$ \\
\hline Kishen et al. ${ }^{23}$ & NPsCS & Ação antimicrobiana & NPs > NPs + Grossman tipo 801 \\
\hline Del Carpio-Perochena et al. ${ }^{24}$ & NPsCS & Ação antimicrobiana & NPs $+\mathrm{Ca}(\mathrm{OH}) 2$, aumentou a ação antimicrobiana \\
\hline Shrestha e Kishen $2^{5}$ & NPsCS & Ação antimicrobiana & $\begin{array}{l}\text { NPs foram afetadas pelos inibidores teciduais do } \\
\text { canal radicular }\end{array}$ \\
\hline DaSilva et al. ${ }^{26}$ & NPsCS & Ação antimicrobiana & $\begin{array}{l}\text { NPs + cimento, independente do tratamento } \\
\text { superficial, inibiu o biofilme }\end{array}$ \\
\hline Shrestha e Kishen ${ }^{27}$ & NPsCS & Ação antimicrobiana & NPs + fotoiniciador, alcançou eficácia antibacteriana \\
\hline Shrestha e Kishen ${ }^{28}$ & NPsCS & Ação antimicrobiana & NPs + fotoiniciador, alcançou eficácia antibacteriana \\
\hline Shrestha et al. ${ }^{6}$ & NPsCS & Dispersão das NPs & Houve penetração das NPs nos túbulos dentinários \\
\hline Shvero et al. ${ }^{29}$ & NPsQPEI & Ação antimicrobiana & $\begin{array}{l}\text { AH Plus + NPs, inibiu o crescimento bacteriano } \\
\text { mesmo após } 4 \text { semanas }\end{array}$ \\
\hline Barros et al. ${ }^{30}$ & NPsQPEI & Ação antimicrobiana & $\begin{array}{l}\text { NPs + AH Plus, e NPs + Pulp Canal Sealer EWT, ação } \\
\text { contra biofilmes }\end{array}$ \\
\hline Barros et al.2 & NPsQPEI & $\begin{array}{l}\text { Ação antimicrobiana/ } \\
\text { mecânicas }\end{array}$ & $\begin{array}{l}\text { NPs + cimentos, alterou as propriedades, aumentou } \\
\text { a penetração do cimento e a ação antimicrobiana }\end{array}$ \\
\hline Abramovitz et al. ${ }^{31}$ & NPsQPEI & Ação antimicrobiana & $\begin{array}{l}\text { NPs + cimentos à base de resina epóxi, produzem um } \\
\text { potente efeito antimicrobiano }\end{array}$ \\
\hline Shvero et al..$^{32}$ & NPsQPEI & Ação antimicrobiana & $\begin{array}{l}\text { NPs + cimentos à base de resina epóxi, desestabiliza- } \\
\text { ção e morte bacteriana }\end{array}$ \\
\hline Beyth et al. ${ }^{33}$ & NPsQPEI & Ação antimicrobiana & $\begin{array}{l}\text { Morte bacteriana por meio de variações de } \mathrm{pH} \text {, sem } \\
\text { causar citotoxicidade }\end{array}$ \\
\hline Barros et al. ${ }^{46}$ & NPsQPEI & $\begin{array}{l}\text { Osteogênicas/ } \\
\text { Citotoxicidade }\end{array}$ & $\begin{array}{l}\text { NPs modulam a proliferação a diferenciação de } \\
\text { células ósseas, sem aumentar a toxicidade }\end{array}$ \\
\hline Abramovitz et al..$^{47}$ & NPsQPEI & $\begin{array}{l}\text { Osteogênicas/ } \\
\text { Citotoxicidade }\end{array}$ & $\begin{array}{l}\text { Não há toxicidade e nem proliferação celular com as } \\
\text { NPs+ AH Plus ou Epiphany ou GuttaFlow }\end{array}$ \\
\hline
\end{tabular}


Continuação da tabela

\begin{tabular}{|c|c|c|c|}
\hline Saghiri et al. ${ }^{40}$ & NPsMTA & Mecânicas & NPs + MTA $=$ MTA convencional \\
\hline Saghiri et al. ${ }^{41}$ & NPsMTA & Mecânicas & NPs + MTA > MTA convencional \\
\hline Saghiri et al. ${ }^{42}$ & NPsMTA & Mecânicas & NPs + MTA > MTA convencional \\
\hline Saghiri et al. ${ }^{43}$ & NPsMTA & Mecânicas & NPs + MTA > MTA convencional \\
\hline Saghiri et al. ${ }^{44}$ & NPsMTA & Mecânicas & NPs + MTA > MTA convencional \\
\hline Zehnder et al..$^{34}$ & NPsBAG & Ação antimicrobiana & $\mathrm{NPs}>\mathrm{Ca}(\mathrm{OH}) 2$ \\
\hline Waltimo et al..$^{35}$ & NPsBAG & Ação antimicrobiana & NPs elevam o pH obtendo efeito antimicrobiano \\
\hline Zehnder et al. ${ }^{36}$ & NPsBAG & Ação antimicrobiana & $\mathrm{Ca}(\mathrm{OH}) 2>\mathrm{NPs}$ \\
\hline Zehnder et $\mathrm{al} .3^{7}$ & NPsBAG & Ação antimicrobiana & $\mathrm{Ca}(\mathrm{OH}) 2>\mathrm{NPs}$ \\
\hline Mohn et al. ${ }^{45}$ & NPsBAG & Mecânicas & $\begin{array}{l}\text { NPs + poliisopreno ou policaprolactona, melhoraram } \\
\text { o vedamento de interfaces }\end{array}$ \\
\hline Shrestha et al. ${ }^{21}$ & NPsZnO & Ação antimicrobiana & NPs $<$ NPs + cimento de óxido de zinco \\
\hline Kishen et al. ${ }^{23}$ & NPsZnO & Ação antimicrobiana & $\begin{array}{l}\text { NPs + cimento de óxido de zinco > cimento de óxido } \\
\text { de zinco }\end{array}$ \\
\hline Rad et al. ${ }^{38}$ & NPsZnO & $\begin{array}{l}\text { Ação antimicrobiana/ } \\
\text { mecânicas }\end{array}$ & $\begin{array}{l}\text { Cimentos de NPs > cimento de óxido de zinco e } \\
\text { eugenol ou cimento } \mathrm{AH} 26\end{array}$ \\
\hline Pagonis et al. ${ }^{8}$ & NPsPLGA & Ação antimicrobiana & $\begin{array}{l}\text { NPs + terapia fotodinâmica, reduziu as colônias } \\
\text { bacterianas }\end{array}$ \\
\hline Haseeb et al. ${ }^{9}$ & NPsCHX & Ação antimicrobiana & $\begin{array}{l}\text { Inibição bacteriana durante períodos mais longos de } \\
\text { tempo }\end{array}$ \\
\hline Fan et al. ${ }^{10}$ & $\mathrm{NPsCHX}$ & $\begin{array}{l}\text { Ação antimicrobiana/ } \\
\text { osteogênicas }\end{array}$ & $\begin{array}{l}\text { As NPs + silicato de cálcio mesoporoso, excelente } \\
\text { atividade antimicrobiana e mineralização }\end{array}$ \\
\hline
\end{tabular}

NPsAg - Nanopartículas de prata; NPsCS - Nanopartículas de quitosana; NPsQPEI - Nanopartículas de polietilenimina de amônio quaternário; NPsMTA - Nanopartículas de MTA; NPsBAG- Nanopartículas de vidro bioativo; NPsZnO - Nanopartículas de óxido de zinco; NPsPLGA - Nanopartículas de PLGA; NPsCHX - Nanopartículas de clorexidina

Dentre as propriedades avaliadas, o efeito antimicrobiano foi o mais estudado resultando em um total de 34 artigos. ${ }^{2,5,8-38}$ A maior parte dos estudos encontraram resultados significativos quanto à atividade antimicrobiana, com efeito equivalente ou superior a outros antimicrobianos. Entretanto, três artigos, ${ }^{5,36,37}$ que compararam a atividade antimicrobiana das nanopartículas com o $\mathrm{Ca}(\mathrm{OH})_{2}$, mostraram piores resultados com a utilização das NPs. Um artigo ${ }^{14}$ teve melhor resultado com o $\mathrm{NaOCl} 2 \%$ e outro ${ }^{25}$ obteve a ação antimicrobiana afetada pelos inibidores teciduais do canal radicular (Tabela 1). Alguns estudos obtiveram ação prolongada das nanopartículas, podendo estar presente mesmo após 90 dias. Tal efeito poderia favorecer a manutenção da descontaminação intracanal. ${ }^{9,12,13}$

Todos os estudos que avaliaram as propriedades mecânicas e estruturais dos materiais com nanopartículas encontraram aumento na rigidez, microdureza e uma menor porosidade, que esta relacionada com aumento da resistência de materiais. . $^{2,36-41}$

Quanto à citotoxicidade, estudos revelaram que as nanopartículas podem ser menos citotóxicas do que $\mathrm{NaOCl}$ e CHX, sendo, inclusive biocompatíveis estando em baixas concentrações. ${ }^{4,46,47,49,50}$ Somente no estudo de Bouillaguet $e t$ al. ${ }^{48}$ que foi verificado um aumento da citotoxicidade com o tempo, nas análises do cimento endodôntico GuttaFlow composto por nanopartículas de prata. Abramovitz et al. ${ }^{47}$ não obtiveram proliferação celular em testes com os cimentos AH Plus, Epiphany e GuttaFlow incorporados com nanopartículas de polietilenimina de amônio quaternário.

Nos estudos que testaram as propriedades osteogênicas, as nanopartículas foram responsáveis pela proliferação à diferenciação de células ósseas, ${ }^{46}$ e no encapsulamento de CHX pelo silicato de cálcio mesoporoso, foi produzida uma

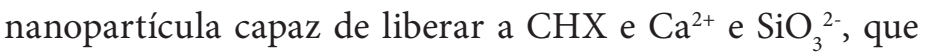
produziram uma mineralização in vitro. ${ }^{10}$

Os nanoencapsulados responsáveis pelo transporte de fármacos para as regiões alvo possuem resultados satisfatórios pois permitem que o fármaco seja protegido e carreado até o local desejado, o que maximiza sua eficácia terapêutica e minimiza os efeitos colaterais indesejáveis. Além disso, é possível controlar a liberação do fármaco aumentado sua biodisponibilidade. ${ }^{8-10}$

Dentre as categorias de nanopartículas avaliadas, como prata, quitosana, polietilenimina de amônio quaternário, agregado trióxido mineral branco, vidro bioativo, óxido de zinco, poli (ácido láctico-co-ácido glicólico) e clorexidina, 
as nanopartículas de prata possuem mais pesquisas publicadas. Já a aplicação de nanopartículas de poli (ácido lácticoco-ácido glicólico) foi encontrada em apenas uma publicação específica para uso endodôntico. As nanopartículas de clorexidina e de prata possuem publicações mais recentes, tendo inclusive pesquisas publicadas em 2017.

As nanopartículas estão em constante progresso. O crescimento atual das pesquisas na área é de grande valia devido à possibilidade de inovação das terapias endodônticas, que visam facilitar e aumentar a qualidade dos procedimentos. O destino dessas investigações está em traduzir técnicas seguras para o desenvolvimento de novos materiais, abordando características específicas como a composição, con- centração, tamanho e morfologia das nanopartículas, nas condições ideais para aplicações. Embora resultados positivos sejam observados com a incorporação de NPs in vitro, estudos clínicos são necessários para ratificar esses achados.

\section{Conclusão}

As nanopartículas estão até o presente momento sendo avaliadas em nível laboratorial, com resultados promissores para práticas clínicas futuras.

\section{Agradecimentos}

Os autores agradecem à Capes (PNPD) e CNPq pelo apoio financeiro.

\section{Referências}

1. Samiei M, Farjami A, Dizaj SM, Lotfipour F. Nanoparticles for antimicrobial purposes in Endodontics: a systematic review of in vitro studies. Mater Sci Eng C. 2016;58:1269-78.

2. Barros J, Silva MG, Rodrigues MA, Alves FR, Lopes MA, Pina-Vaz I, et al. Antibacterial, physicochemical and mechanical properties of endodontic sealers containing quaternary ammonium polyethylenimine nanoparticles. Int Endod J. 2014;47(8):725-34

3. Shrestha A, Kishen A. Antibacterial nanoparticles in Endodontics: a review. J Endod. 2016;42(10):1417-26.

4. Abbaszadegan A, Nabavizadeh M, Gholami A, Aleyasin ZS, Dorostkar S, Saliminasab M, et al. Positively charged imidazolium-based ionic liquid-protected silver nanoparticles: a promising disinfectant in root canal treatment. Int Endod J. 2015;48(8):790-800.

5. Alabdulmohsen ZA, Saad AY. Antibacterial effect of silver nanoparticles against Enterococcus faecalis. Saudi Endod J. 2017;7:29-35.

6. Shrestha A, Fong SW, Khoo BC, Kishen A. Delivery of antibacterial nanoparticles into dentinal tubules using high-intensity focused ultrasound. J Endod. 2009;35(7):1028-33.

7. Jadhav R, Bhide S, Prasad BLV, Kunchiraman B, Shimpi J, Nandhini U. Silver nanoparticles: a new perspective in endodontic therapy. IIOABJ. 2016;7(6):77-81. 8. Pagonis TC, Chen J, Fontana CR, Devalapally H, Ruggiero K, Song X, et al. Nanoparticle-based endodontic antimicrobial photodynamic therapy. J Endod. 2010;36(2):322-8.

9. Haseeb R, Lau M, Sheah M, Montagner F, Quiram G, Palmer K, et al. Synthesis and characterization of new chlorhexidine-containing nanoparticles for root canal disinfection. Materials. 2016;9(6):452.

10. Fan W, Li Y, Sun Q, Ma T, Fan B. Calcium-silicate mesoporous nanoparticles loaded with chlorhexidine for both anti-Enterococcus faecalis and mineralization properties. J Nanobiotechnol. 2016;14(1):72.

11. Lotfi M, Vosoughhosseini S, Ranjkesh B, Khani S, Saghiri M, Zand V. Antimicrobial efficacy of nanosilver, sodium hypochlorite and chlorhexidine gluconate against Enterococcus faecalis. Afr J Biotechnol. 2011;10(35):6799-803.

12. Moghadas L, Shahmoradi M, Narimani T. Antimicrobial activity of a new nanobased endodontic irrigation solution: in vitro study. Dent Hypotheses. 2012;3(4):142-46.

13. González-Luna P-IV, Martínez-Castañón G-A, Zavala-Alonso N-V, Patiño -Marin N, Niño-Martínez N, Morán-Martínez J, et al. Bactericide effect of silver nanoparticles as a final irrigation agent in endodontics on Enterococcus faecalis: an ex vivo study. J of Nanomaterials. 2016;1-7.

14. Wu D, Fan W, Kishen A, Gutmann JL, Fan B. Evaluation of the antibacterial efficacy of silver nanoparticles against Enterococcus faecalis biofilm. J Endod. 2014;40(2):285-90.

15. Bo D, Kayombo CM. Effect of nanosilver gel, chlorhexidine gluconate, and camphorated phenol on Enterococcus faecalis biofilm. Int Sch Res Notices. 2014;1-5.

16. Bruniera JFB, Corrêa Silva-Sousa YTC, Lara MG, Pitondo-Silva A, Marcaccini AM, Miranda CES. Development of intracanal formulation containing silver nanoparticles. Braz Dent J. 2014;25(4):302-06.
17. Javidi M, Afkhami F, Zarei M, Ghazvini K, Rajabi O. Efficacy of a combined nanoparticulate/calcium hydroxide root canal medication on elimination of Enterococcus faecalis. Aust Endod J. 2014;40:61-65.

18. Afkhamia F, Pourhashemib SJ, Sadegha M, Salehic Y, Fardd MJK. Antibiofilm efficacy of silver nanoparticles as a vehicle for calcium hydroxide medicament against Enterococcus faecalis. J Dent. 2015;43(12):1573-9.

19. Samiei M, Aghazadehb M, Lotfia M, Shakoeia S, Aghazadehc Z, Pakdeld SMV. Antimicrobial efficacy of mineral trioxide aggregate with and without silver nanoparticles. Iran Endod J. 2013;8(4):166-70.

20. Shantiaee Y, Maziar F, Dianat O, Mahjour F. Comparing microleakage in root canals obturated with nanosilver coated gutta-percha to standard gutta-percha by two different methods. Iran Endod J. 2011;6(4):140-45.

21. Shrestha A, Shi Z, Neoh KG, Kishen A. Nanoparticulates for antibiofilm treatment and effect of aging on its antibacterial activity. J Endod. 2010;36(6):1030-5. 22. Del Carpio-Perochena A, Kishen A, Shrestha A, Bramante CM. Antibacterial properties associated with chitosan nanoparticle treatment on root dentin and 2 types of endodontic sealers. J Endod. 2015;41(8):1353-8.

23. Kishen A, Shi Z, Shrestha A, Neoh KG. An investigation on the antibacterial and antibiofilm efficacy of cationic nanoparticulates for root canal disinfection. J Endod. 2008;34(12):1515-20.

24. Del Carpio-Perochena A, Kishen A, Felitti R, Bhagirath AY, Medapati MR, Lai C, et al. Antibacterial properties of chitosan nanoparticles and propolis associated with calcium hydroxide against single- and multispecies biofilms: an in vitro and in situ study. J Endod. In press 2017.

25. Shrestha A, Kishen A. The effect of tissue inhibitors on the antibacterial activity of chitosan nanoparticles and photodynamic therapy. J Endod 2012;38(9):1275-78.

26. DaSilva L, Finer Y, Friedman S, Basrani B, Kishen A. Biofilm formation within the interface of bovine root dentin treated with conjugated chitosan and sealer containing chitosan nanoparticles. J Endod. 2013;39(2):249-53.

27. Shrestha A, Kishen A. Antibacterial efficacy of photosensitizer functionalized biopolymeric nanoparticles in the presence of tissue inhibitors in root canal. J Endod. 2014;40(4):566-70.

28. Shrestha A, Kishen A. Antibiofilm efficacy of photosensitizer-functionalized bioactive nanoparticles on multispecies biofilm. J Endod. 2014;40(10):1604-10. 29. Shvero DK, Abramovitz I, Zaltsman N, Davidi MP, Weiss EI, Beyth N. Towards antibacterial endodontic sealers using quaternary ammonium nanoparticles. Int Endod J. 2013;46(8):747-54.

30. Barros J, Silva MG, Rôças IN, Gonçalves LS, Alves FF, Lopes MA, et al. Antibiofilm effects of endodontic sealers containing quaternary ammonium polyethylenimine nanoparticles. J Endod. 2014;40(8):1167-71.

31. Abramovitz I, Wisblech D, Zaltsman N, Weiss EI, Beyth N. Intratubular antibacterial effect of polyethyleneimine nanoparticles: an ex vivo study in human teeth. J of Nanomaterials. 2015;1-5.

32. Shvero DK, Zaltsman N, Weiss EI, Polak D, Hazan R, Beyth N. Lethal bacterial trap: cationic surface for endodontic sealing. J Biomed Mater Res A. 2016;104(2):427-34.

33. Beyth N, Shvero DK, Zaltsman N, Houri-Haddad Y, Abramovitz I, Davidi 
MP, et al. Rapid kill-novel endodontic sealer and Enterococcus faecalis. PLoS ONE. 2013;8(11):1-10.

34. Zehnder M, Söderling E, Salonen J, Waltimo T. Preliminary evaluation of bioactive glass s53p4 as an endodontic medication in vitro. J Endod. 2004;30(4):220-24. 35. Waltimo T, Brunner TJ, Vollenweider M, Stark WJ, Zehnder M. Antimicrobial effect of nanometric bioactive glass 45S5. J Dent Res. 2007;86(8):754-7. 36. Zehnder M, Luder HU, Schätzle M, Kerosuo E, Waltimo T. A comparative study on the disinfection potentials of bioactive glass S53P4 and calcium hydroxide in contra-lateral human premolars ex vivo. Int Endod J. 2006;39(12):952-8. 37. Zehnder M, Baumgartner G, Marquardt K, Paqué F. Prevention of bacterial leakage through instrumented root canals by bioactive glass $\mathrm{S} 53 \mathrm{P} 4$ and calcium hydroxide suspensions in vitro. Oral Surg Oral Med Oral Pathol Oral Radiol Endod. 2007;103(3):423-8.

38. Rad MS, Kompany A, Zak AK, Javidi M. Mortazavi SM. Microleakage and antibacterial properties of $\mathrm{ZnO}$ and $\mathrm{ZnO}$ :Ag nanopowders prepared via a sol-gel method for endodontic sealer application. J Nanopart Res. 2013;15: 1925-6.

39. Nevarez-Rasconb A, Orrantia-Borundaa E, González-Hernándeza J, FloresGallardoa S, Hurtado-Macías A. Mechanical characterization of optical glass fiber coated with a thin film of silver nanoparticles by nanoindentation. Mat Letters. 2014;136:63-66.

40. Saghiri MA, Asatourian A, Orangi J, Lotfi M, Soukup JW, Garcia-Godoy F, Sheibani N. Effect of particle size on calcium release and elevation of $\mathrm{pH}$ of endodontic cements. Dent Traumatol. 2015;31(3):196-201.

41. Saghiri MA, Asatourian A, Orangi J, Soukup JW, Gutmann JL, Garcia-Godoy $\mathrm{F}$, Sheibani N. The influence of antibiotics on the physical properties of endodontic cements. Giornale Italiano di Endodonzia. 2016;30:89-95.

42. Saghiri MA, Asgar K, Lotfi M, Garcia-Godoy F. Nanomodification of mi- neral trioxide aggregate for enhanced physiochemical properties. Int Endod J. 2012;45(11):979-88.

43. Saghiri MA, Godoy FG, Gutmann JL, Lotfi M, Asatourian A, Sheibani N, Elyasi $\mathrm{M}$. The effect of $\mathrm{pH}$ on solubility of nano-modified endodontic cements. J Conserv Dent. 2014;17(1):13-7.

44. Saghiri MA, Nazari A, Garcia-Godoy F, Asatourian A, Malekzadeh M, Elyasi M. Mechanical response of dental cements as determined by nanoindentation and scanning electron microscopy. Microsc Microanal. 2013;19(6):1-7.

45. Mohn D, Bruhin C, Luechinger NA, Stark WJ, Imfeld T, Zehnder M. Composites made of flame-sprayed bioactive glass $45 \mathrm{~S} 5$ and polymers: bioactivity and immediate sealing properties. Int Endod J. 2010;43(11):1037-46.

46. Barros J, Costa-Rodrigues J, Lopes MA, Pina-Vaz I, Fernandes MH. Response of human osteoblastic and osteoclastic cells to AH Plus and Pulp Canal Sealer containing quaternary ammonium polyethylenimine nanoparticles. J Endod. 2014;40(8):1149-55.

47. Abramovitz I, Beyth N, Weinberg G, Borenstein A, Polak D, Kesler-Shvero $D$, et al. In vitro biocompatibility of endodontic sealers incorporating antibacterial nanoparticles. J of Nanomaterials. 2012;1-9.

48. Bouillaguet S, Wataha JC, Tay FR, Brackett MG, Lockwood PE. Initial in vitro biological response to contemporary endodontic sealers. J Endod. 2006;32(10):989-92.

49. Gomes-Filho JE, Silva FO, Watanabe S, Cintra LTA, Tendoro KV, Dalto LG, Pacanaro SV, Lodi CS, de Melo FFF. Tissue reaction to silver nanoparticles dispersion as an alternative irrigating solution. J Endod. 2010;36:1698-702. 50. Chan EL, Zhang C, Cheung GS. Cytotoxicity of a novel nano-silver particle endodontic irrigant. Clin Cosmet Investig Dent. 2015;7:65-74.

\section{Mini Currículo e Contribuição dos Autores}

1. Iris Sol Figueiredo Telles - cirurgiã-dentista. Contribuição: levantamento dos artigos e redação do artigo.

2. Maíra do Prado - cirurgiã-dentista e PhD. Contribuição: participação científica e intelectual efetiva para a redação do artigo, revisão crítica e aprovação final.

3. Renata Antoun Simão - física e PhD. Contribuição: participação científica e intelectual efetiva para a redação do artigo e revisão crítica.

Recebido em: 10/04/2017 / Aprovado em: 13/06/2017

Autora Correspondente

Maíra do Prado

E-mail: mairapr@hotmail.com 